\title{
Calendar of
}

Orlando, FL

Orlando, FL

Orlando, FL

Orlando, FL

Orlando, FL

Orlando, FL

Orlando, FL

Orlando, FL

Orlando, FL

Orlando, FL

Orlando, FL

Orlando, FL

Orlando, FL

Orlando, FL

Orlando, FL

Orlando, FL
13-17 January

12-13 January

12-13 January

13 January

13 January

13 January

13 January

12-13 January

13 January

13 January

14 January

15 January

13-17 January

13-17 January

13-17 January

13-17 January 82nd AMS Annual Meeting

AMS Short Course on Neural Network Applications to Environmental Sciences

AMS Short Course on the Fundamentals of Doppler and Polarimetric Weather Radar

AMS Short Course on Verification of Climate Forecasts

AMS Short Course on Land-Surface-Climate Interaction

AMS Short Course on QPF and QPE: Quantitative Precipitation Forecasting and Quantitative Precipitation Estimation

AMS Weather Entrepreneur Workshop

First AMS Student Conference and Career Fair

Special Conference on African Climate and Environment

Communication Workshop

Atlas Symposium: A Tribute to David Atlas

A Half Century of Progress in Meteorology: The Richard Reed Symposium

Symposium on Observations, Data Assimilation, and Probabilistic Prediction

Third Symposium on Environmental Applications: Facilitating the Use of Environmental Information

18th International Conference on Interactive Information and Processing Systems (IIPS) for Meteorology,

Oceanography, and Hydrology

16th Conference on Probability and Statistics in the Atmospheric Sciences

"New listing in Calendar, *Meeting administered by AMS, + New listing — see Announcements/Calls for Papers 


\section{AMERICAN METEOROLOGICAL SOCIETY}

\section{Calendar

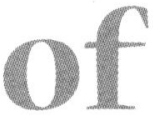

Orlando, FL

Orlando, FL

Orlando, FL

Orlando, FL

Orlando, FL

Orlando, FL

San Diego, CA

Portland, OR

Portland, OR

Norfolk, VA

Norfolk, VA

Norfolk, VA

Ogden, UT

Ogden, UT

Park City, UT

Williamsburg, VA

Wageningen,

The Netherlands

San Antonio, TX
13-17 January

13-17 January

13-17 January

13-17 January

13-17 January

13-17 January

29 April-3 May

13-16 May

13-16 May

20-24 May

20-24 May

20-24 May

3-7 June

3-7 June

17-21 June

23-27 June

15-19 July

12-16 July 16th Conference on Hydrology

13th Symposium on Global Change and Climate Variations

11th Symposium on Education

Sixth Symposium on Integrated Observing Systems

Fourth Conference on Atmospheric Chemistry: Urban, Regional, and Global Scale Impacts of Air Pollutants

Interactive Symposium on the Advanced Weather Interactive Processing System (AWIPS)

25th Conference on Hurricanes and Tropical Meteorology

13th Conference on Applied Climatology

10th Conference on Aviation, Range, and Aerospace Meteorology

25th Conference on Agricultural and Forest Meteorology

12th Joint Conference on the Applications of Air Pollution Meteorology with the Air and Waste Management Association (A\&WMA)

Fourth Symposium on the Urban Environment

11th Conference on Atmospheric Radiation

11th Conference on Cloud Physics

10th Conference on Mountain Meteorology

31st Conference on Broadcast Meteorology

15th Symposium on Boundary Layers and Turbulence

19th Conference on Weather Analysis and Forecasting/15th Conference on Numerical Weather Prediction 


\title{
AMERICAN METEOROLOGICAL SOCIETY
}

\section{Calendar

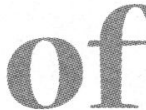

San Antonio, TX

San Antonio, TX

Long Beach, CA

Wellington,

New Zealand

Worcester, MA

Tempe, AZ

Indore, M.P., India

Seattle, WA

Issy-les-Moulineaux

France

New Orleans, LA

New London, CT

Rome, Italy

Quebec City,

PQ, Canada

Honolulu, HI
12-16 July

21st Conference on Severe Local Storms

4-8 November

${ }^{\#+} 12$ th Conference on Middle Atmosphere

\section{AMS 2003 Meetings}

\author{
9-13 February \\ ${ }^{\#+83 n d ~ A M S ~ A n n u a l ~ M e e t i n g ~}$
}

24-28 March

Seventh International Conference on Southern Hemisphere Meteorology and Oceanography

\section{Other 2001 Meetings of Interest}

30 Nov.-1 Dec. Second Southern New England Weather Conference

5-8 December Third International Symposium on Environmental Hydraulics with a Special Theme in Urban Fluid Dynamics

16-18 December International Congress of Chemistry and Environment

\section{Other 2002 Meetings of Interest}

1-2 March

8-12 March

13-17 May

19-22 June

24-28 June

7-12 July

22-26 July
\#+Pacific Northwest Weather WorkshopC2001

${ }^{\#+}$ 12th International Weather Festival

Mississippi River Climate and Hydrology Conference

Council on Undergraduate Research National Conference

11th Symposium on Acoustic Remote Sensing (ISARS2002)

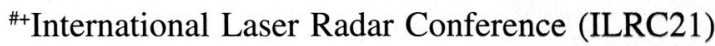

International Tropical Rainfall Measuring Mission (TRMM) Science Conference

"New listing in Calendar, "Meeting administered by AMS, + New listing - see Announcements/Calls for Papers 


\section{AMERICAN METEOROLOGICAL SOCIETY}

Reading, Berks.,

United Kingdom

Rhodes, Greece

Rhodes, Greece

Tucson, AZ
2-6 September

The World Weather Research Programme's (WWRP) International Conference on Quantitative Precipitation Forecasting (QPF)

16-18 September

Fourth International Conference on Environmental Problems in Coastal Regions: COASTAL ENVIRONMENT 2002

17-19 September

Third International Conference on Oil \& Hydrocarbon Spills, Modleling, Analysis \& Control: OIL SPILL 2002

18-20 September Third International Conference on Management, Operation, Design and Building of Ports, Marinas and other Maritime Works, PORTS \& MARINAS 2002

16-18 October International Lightning Detection Conference 